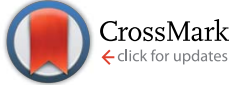

Cite this: RSC Adv., 2017, 7, 1282

Accepted 15th November 2016

DOI: $10.1039 / \mathrm{c} 6 \mathrm{ra25470e}$

www.rsc.org/advances

\section{Three-dimensional network of coaxial carbon nanotube/manganese oxides electrode for supercapacitors $\uparrow$}

\author{
S. J. Cho,,$^{a}$ R. Chen, $\dot{t}^{a}$ M. Lee, ${ }^{b}$ Y. S. Kang, ${ }^{c}$ S. Lee ${ }^{d}$ and H. Lee
}

A three-dimensional network of carbon nanotubes (3DNC) coaxially coated with manganese oxides $\left(\mathrm{MnO}_{x}\right)$ is used as an electrode for supercapacitors. 3DNC serves as a stable and conductive framework for controlled electrochemical deposition (ECD), and provides sufficient voids for fast ionic transport and diffusion. The coated thin layer $\mathrm{MnO}_{x}$ reduces ion diffusion and electron transport distance, enabling fast reversible faradic reactions.
As one of the most promising electrochemical energy storage materials, manganese oxides have been studied extensively as supercapacitor electrode materials due to their low cost, nontoxicity and theoretically high capacity of about $1370 \mathrm{~F} \mathrm{~g}^{-1} \cdot{ }^{1-10}$ In particular, low dimensional nanostructured $\mathrm{MnO}_{x}$ with different morphologies has been developed as an electrode material and showed its advantages in charge storage capability. ${ }^{4,9,11-15}$ However, its intrinsic poor electrical conductivity is a current drawback to utilize $\mathrm{MnO}_{x}$ as a high-rate performance supercapacitor. ${ }^{1,3-5,7}$ To overcome this problem, an effective way is to incorporate $\mathrm{MnO}_{x}$ with other metal elements $(\mathrm{Ni}, \mathrm{Ru}, \mathrm{Al}, \mathrm{Sn} \text { and } \mathrm{Pb} \text { etc. })^{6,7}$ or porous, high surface area and conductive nanostructures such as carbonaceous sphere, ${ }^{16}$ carbon nanotubes, ${ }^{17}$ graphene ${ }^{18}$ and conductive polymers. ${ }^{19}$ Among those for advanced metal oxide-based hybrid nanostructure design, coaxial thin layer structure with ordered nanostructure arrays is considered as one of promising approaches for achieving the goal. Previous research has shown only a very thin layer of metal oxide material (several tens of nanometers from the surface) participated in the redox reaction. ${ }^{20}$ Hence, coaxial thin layer structure could allow cations to intercalate into entire active materials and shorten electron transport distance inside the active materials. Yamauchi Y. et al. ${ }^{17}$ reported on the development of multi-

\footnotetext{
a'Department of Chemistry, Hanyang University, Seoul 04763, Korea. E-mail: haiwon@ hanyang. ac. $k r$

${ }^{b}$ Center for Nanobio Integration \& Convergence Engineering, National Nanofab Center, Daejeon 34141, Korea

'Department of Energy Engineering, Hanyang University, Seoul 04763, Korea

${ }^{d}$ Department of Advanced Materials Engineering, University of Suwon, Gyeonggi-do 18323, Korea

'Institute of Nano Science and Technology, Hanyang University, Seoul 04763, Korea $\dagger$ Electronic supplementary information (ESI) available. See DOI: 10.1039/c6ra25470e

¥S. J. Cho and R. Chen contributed equally to this work.
}

walled carbon nanotube-manganese oxide core-shell structures utilized for asymmetric supercapacitor applications which shows a specific capacitance $\left(C_{\mathrm{sp}}\right)$ of $185 \mathrm{~F} \mathrm{~g}^{-1}$ at a scan rate of $5 \mathrm{mV} \mathrm{s}^{-1}$. Ordered nanostructure array is also an excellent candidate in aspect of providing a conductive path way not only for electron transport but also for resolving the ionic diffusion and transportation issues of supercapacitor electrodes. Zhu S. J. et al. ${ }^{21}$ reported the development of coaxial mesoporous manganese dioxide/amorphous carbon nanotubes arrays for advanced asymmetric supercapacitors which exhibit the optimized pseudocapacitance performance (362 F $\mathrm{g}^{-1}$ ) with good cycling stability, and ideal rate capability. Ionic diffusion and transportation are critical points to improve superior energy density, power density and charge efficiency due to shorter diffusion length in void volume. ${ }^{22,23}$ Longer life cycle and stronger volume retention during the charge and discharge process can be obtained through the nanostructures. Here, we demonstrate a coaxial thin layer $\mathrm{MnO}_{x}$ hybrid nanostructure array for high performance $\mathrm{MnO}_{x}$ supercapacitors.

In our previous research works, three-dimensional network of carbon nanotubes (3DNC) on pillar structures have been developed. ${ }^{24-29}$ As demonstrated, 3DNC has a hierarchically arrayed structure with interconnected CNTs between ordered arrays of Si pillars. ${ }^{28}$ Because of its unique structural properties, 3DNC has been applied to many applications such as catalysis, ${ }^{26}$ colorimetric sensor, ${ }^{25}$ gas sensor, ${ }^{27}$ strain sensor, ${ }^{30}$ microfluidic platform, ${ }^{28}$ cell seeding platform, ${ }^{24}$ and surface-enhanced Raman scattering. ${ }^{29,31}$ The CNTs interconnected between ordered Si pillars are stable during electrochemical reactions and the 3DNC structure provides controlled voids for ionic diffusion onto the surface of active materials. It would be significant to study the capacitance performance by incorporating energy storage materials like $\mathrm{MnO}_{x}$. 
In this work, $3 \mathrm{DNC}$ coaxially coated with $\mathrm{MnO}_{x}$ is used as an electrode for supercapacitor. The 3DNC structure is not only served as a stable and conductive framework for controlled electrochemical deposition (ECD) of $\mathrm{MnO}_{x}$ and a current collector for charge/discharge, but also provides sufficient voids for fast ionic transport between CNTs and ionic diffusion onto the surface of active materials. Simultaneously, the coated thin layer $\mathrm{MnO}_{x}$ reduces the distance of ion diffusion and electron transport, enabling fast and reversible faradic reactions.

The schematic diagram of a fabrication process of 3DNC/ $\mathrm{MnO}_{x}$ and its corresponding scanning electron microscope (SEM) images are presented in Fig. 1. Amorphous carbon nanotubes on bare Si wafer (2DNC) was synthesized under the same condition for comparison and their SEM images are shown in Fig. S1. $\dagger$ The pristine Si pillars shown in Fig. 1a have a diameter of $1.0 \mu \mathrm{m}$ and height of $3.0 \mu \mathrm{m}$, respectively. Fig. $1 \mathrm{~b}$ describes that most CNTs were tangled on both the sides and tops of pillars, and some CNTs were suspended between pillars after synthesizing CNTs. The insert image of TEM in Fig. 1b verifies the multi-walled carbon nanotube grown on the Si pillar structure with average diameter of $25.1 \mathrm{~nm}$. Before coating $\mathrm{MnO}_{x}$, a thin layer of Pt was firstly coated on the synthesized 3DNC using ion sputter. The Pt plays an important role in combining CNTs and target $\mathrm{MnO}_{x}$. As demonstrated in previous work, ${ }^{26} \mathrm{Pt}$ coating is an essential step to improve the mechanical strength and maintaining the morphology of the 3DNC structures as well as enhancing electron transportation and adhesion between 3DNC and $\mathrm{MnO}_{x}$. Fig. 1c shows the SEM image of 3DNC coated with $\mathrm{MnO}_{x}$. After 3 min deposition of $\mathrm{MnO}_{x}$, the original 3D network morphology is still preserved and the diameter of CNTs is increased obviously. The inset TEM image clearly presents CNTs as a backbone were well coated with Pt and another thin layer which is composed of amorphous $\mathrm{MnO}_{x}$ with a thickness of 5-10 $\mathrm{nm}$. The average diameter of the CNT and $\mathrm{MnO}_{x}$ coaxial composite bundles is around $43.1 \mathrm{~nm}$. EDX elemental mapping is introduced for confirming the coaxial structure. In Fig. 2a a $3 \mathrm{DNC} / \mathrm{MnO}_{x}$ bundle is selected as the mapping area and then $\mathrm{O}, \mathrm{C}, \mathrm{Pt}$ and $\mathrm{Mn}$ elements are scanned. Fig. $2 \mathrm{~b}$ and c clearly show that $\mathrm{Mn}$ and $\mathrm{Pt}$ elements are distributed at the bundle outside layer and inner core,

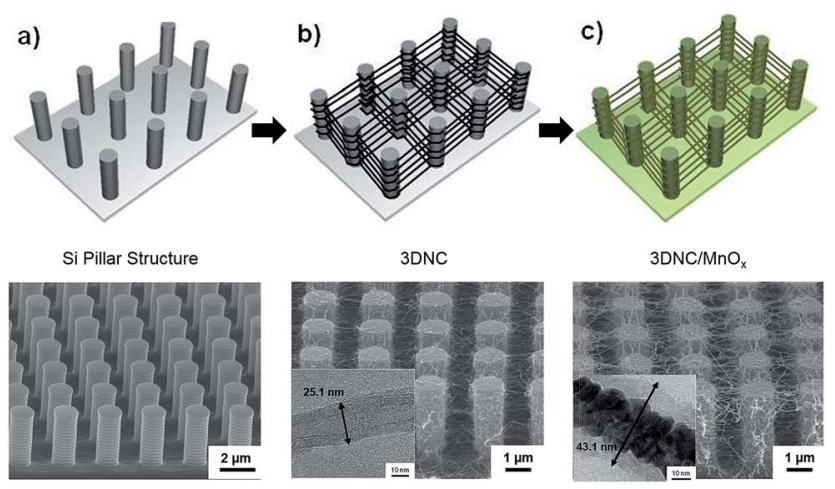

Fig. 1 Schematic illustration of $3 \mathrm{DNC} / \mathrm{MnO}_{x}$ fabrication process and SEM images of (a) pristine Si pillar substrate, (b) as synthesized 3DNC and (c) 3DNC/MnO${ }_{x}$.
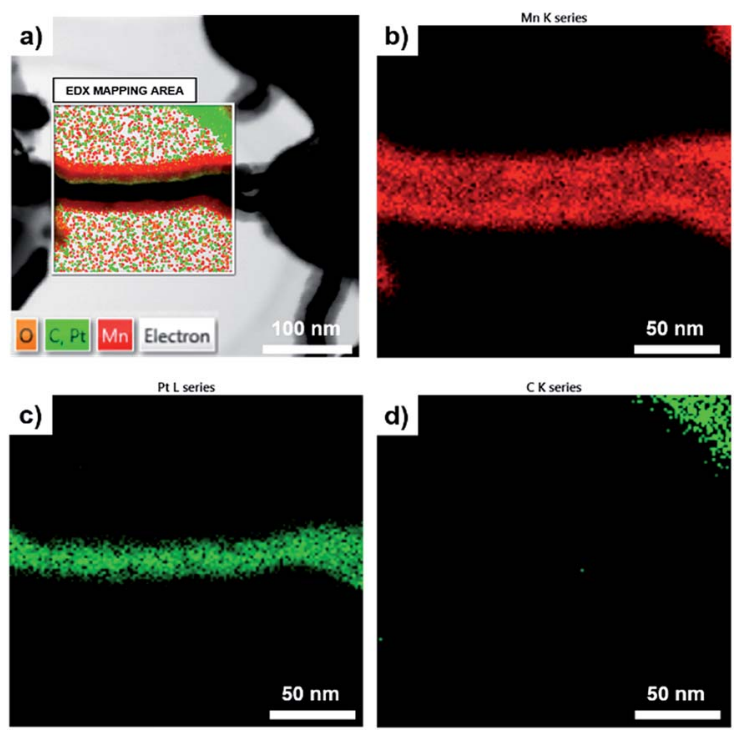

Fig. 2 (a) EDX elemental mapping of 3DNC/MnO${ }_{x}$ coaxial bundle; (b) Mn element, (c) Pt element and (d) C element mapping at the selected area.

respectively. However, $\mathrm{C}$ element from $\mathrm{CNT}$ is hardly to be detected in the bundle as shown in Fig. $2 \mathrm{~d}$. The reason could be that the covered Pt layer blocks or disturbs the signals from inner CNT. By comparing different $\mathrm{MnO}_{x}$ deposition time, 3 min coating shows best performance. Details are explained in Fig. S2 and S3.†

For a better understanding of the chemical composition and oxidation state of $\mathrm{Mn}, 3 \mathrm{DNC} / \mathrm{MnO}_{x}$ was characterized by X-ray photoelectron spectroscopy (XPS) as shown in Fig. 3a. Peaks of Mn 2p, O 1s, and C 1s are observed and the insert shows two peaks located at $654.2 \mathrm{eV}$ and $642.6 \mathrm{eV}$, which are attributed to Mn 2 $\mathrm{p}_{1 / 2}$ and $\mathrm{Mn} 2 \mathrm{p}_{3 / 2}$, respectively. Those results are in good agreement with previously reported data., ${ }^{1,2,32}$ Beyond coaxial and uniform coating, the oxidation state and electrochemical performance of $\mathrm{MnO}_{x}$ can be controlled by changing pulse intervals in deposition. The pseudocapacitance value of $\mathrm{MnO}_{x}$ is affected by $\mathrm{Mn}$ oxidation number. Since one alkali ion reacts with one $\mathrm{MnO}_{x}$ molecule, $\mathrm{MnO}_{2}$ (Iv) has the highest ideal pseudocapacitance $\left(1370 \mathrm{~F} \mathrm{~g}^{-1}\right)$. Because $\mathrm{MnO}_{x}$ is produced by reducing a permanganate ion in this experiment, the deposition of $\mathrm{MnO}_{x}$ with higher oxidation number requires lower voltage. ${ }^{33}$ During constant current deposition, the applied voltage increases automatically as deposition time increases. This is because permanganate ion consumption occurs near the electrode, and higher voltage is required to deposit $\mathrm{MnO}_{x}$ constantly. To refill consumed ions, the ECD process is carried out with intervals of zero current for free diffusion of permanganate ions. Ion diffusion is more considerable for threedimensional structures due to larger diffusion voids. Three samples are prepared with $1 \mathrm{~s}$ deposition time and diffusion times of 15, 30, $60 \mathrm{~s}$, per cycle. The oxidation state of $\mathrm{MnO}_{x}$ is calculated using the peak height ratio of XPS spectra. The oxidation number of deposited $\mathrm{MnO}_{x}$ is determined by the peak height ratio of $\mathrm{Mn} 2 \mathrm{p}_{3 / 2}$ versus $\mathrm{O} 2 \mathrm{p}$ (Table $\mathrm{S} 1 \dagger$ ). The peak height 
a)
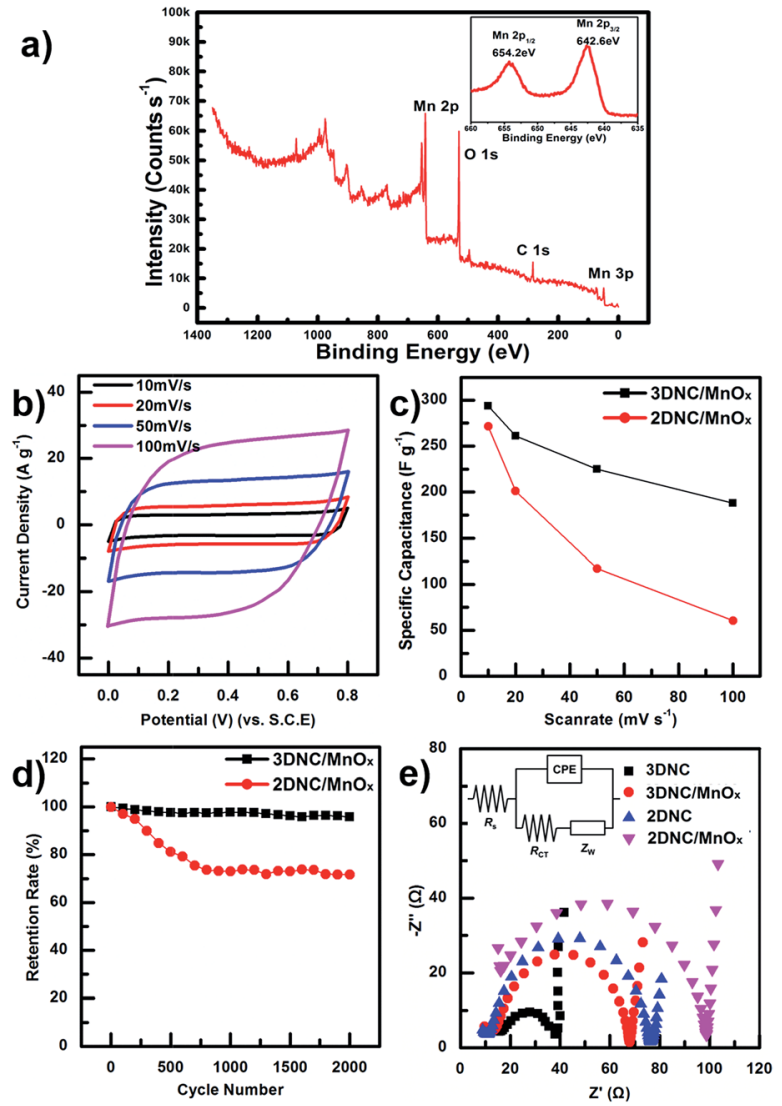

Fig. 3 (a) XPS spectrum of $3 \mathrm{DNC} / \mathrm{MnO}_{x}$; (b) $\mathrm{CV}$ curves of $3 \mathrm{DNC} / \mathrm{MnO}_{x}$ at scan rate of $10,20,50$ and $100 \mathrm{mV} \mathrm{s}^{-1}$ in $1 \mathrm{M} \mathrm{Na}_{2} \mathrm{SO}_{4}$ solution; (c) $C_{\text {sp }}$ of $3 \mathrm{DNC} / \mathrm{MnO}_{x}$ and $2 \mathrm{DNC} / \mathrm{MnO}_{x}$ at different scan rates; (d) cycling stability of 3DNC/MnO and $2 \mathrm{DNC} / \mathrm{MnO}_{x}$ at scan rate of $100 \mathrm{mV} \mathrm{s}^{-1}$ for 2000 cycles; (e) Nyquist plots of 3DNC, 3DNC/MnO, $2 \mathrm{DNC}$, and $2 \mathrm{DNC} / \mathrm{MnO}_{\mathrm{x}}$.

ratios of each sample are 1.03, 1.01 and 0.99 (Fig. S4 $\dagger$ ), with $C_{\mathrm{sp}}$ of 190,248 and $294 \mathrm{~F} \mathrm{~g}^{-1}$, respectively, at a scan rate of $10 \mathrm{mV}$ $\mathrm{s}^{-1}$. Thus, $60 \mathrm{~s}$ diffusion time shows the best $C_{\mathrm{sp}}$ value. While the peak height ratio approaching 0.95, more and more manganese dioxide is produced and the pseudo-capacitance of $\mathrm{MnO}_{x}$ could be improved. ${ }^{34}$

To verify the electrochemical performance of $3 \mathrm{DNC} / \mathrm{MnO}_{x}$, $\mathrm{CV}$ was conducted. The CV curves of $3 \mathrm{DNC} / \mathrm{MnO}_{x}$ (Fig. $3 \mathrm{~b}$ ) are closer to a rectangular shape and a mirror image at each scan rate than those of $2 \mathrm{DNC} / \mathrm{MnO}_{x}$ (Fig. $\mathrm{S} 1 \mathrm{c} \dagger$ ). For $3 \mathrm{DNC} / \mathrm{MnO}_{x}$, the shape of $\mathrm{MnO}_{x} \mathrm{CV}$ curves did not change significantly with the increase of scan rate, indicating the better capacitive behavior. Fig. 3c shows $C_{\mathrm{sp}}$ values at different scan rates. The calculated $C_{\text {sp }}$ values of $3 \mathrm{DNC} / \mathrm{MnO}_{x}$ are 294, 261, 225 and $188 \mathrm{~F} \mathrm{~g}^{-1}$ at scan rates of $10,20,50$ and $100 \mathrm{mV} \mathrm{s}^{-1}$, respectively, which are larger than those of $2 \mathrm{DNC} / \mathrm{MnO}_{x}$ at the same scan rate (Fig. $\mathrm{S} 1 \mathrm{c} \dagger$ ). At low scan rate $10 \mathrm{mV} \mathrm{s}^{-1}$, the $C_{\mathrm{sp}}$ values of these two materials show a small difference. However, when scan rate increases, the $C_{\text {sp }}$ value of $2 \mathrm{DNC} / \mathrm{MnO}_{x}$ is decreased more dramatically than $3 \mathrm{DNC} / \mathrm{MnO}_{x}$. By comparing the SEM images of $3 \mathrm{DNC} / \mathrm{MnO}_{x}$ (Fig. 1c), the CNTs in $2 \mathrm{DNC} / \mathrm{MnO}_{x}$ (Fig. S1a $\dagger$ ) are tightly connected or touched with adjacent ones. In this case, the large amount of voids obtained in $3 \mathrm{DNC} / \mathrm{MnO}_{x}$ structure promoted
Table 1 Fitting values of the equivalent circuit elements of faradic charge transfer resistance $\left(R_{c t}\right)$ and electrolyte resistance $\left(Z_{\mathrm{w}}\right)$

\begin{tabular}{lllll}
\hline \multirow{4}{*}{ Parameter } & \multicolumn{2}{l}{ Fitting value $[\Omega]$} & \\
\cline { 2 - 5 } & $3 \mathrm{DNC}$ & $3 \mathrm{DNC} / \mathrm{MnO}_{x}$ & 2DNC & 2DNC/MnO \\
\hline$R_{\mathrm{ct}}$ & 23.6 & 54.9 & 65.5 & 82.8 \\
$Z_{\mathrm{w}}$ & 11.7 & 11.9 & 15.3 & 20.3 \\
\hline
\end{tabular}

the ion diffusion and transfer speed between electrolyte and electrode and finally the capacitive performance was improved.

The cycle stability was confirmed for 2000 cycles at scan rate of $100 \mathrm{mV} \mathrm{s}{ }^{-1}$. Fig. 3d shows the life cycle test of $3 \mathrm{DNC} / \mathrm{MnO}_{x}$ and $2 \mathrm{DNC} / \mathrm{MnO}_{x}$. The $C_{\mathrm{sp}}$ of $3 \mathrm{DNC} / \mathrm{MnO}_{x}$ retains about $96 \%$ of its initial capacitance even after 2000 cycles while $2 \mathrm{DNC} / \mathrm{MnO}_{x}$ retains only $72 \%$. Fig. S5 $\uparrow$ compares the morphology of 3DNC/ $\mathrm{MnO}_{x}$ coaxial bundles before and after 2000 cycles test. There are no significant change could be observed except after life cycle test the $3 \mathrm{DNC} / \mathrm{MnO}_{x}$ surface becomes rougher which could be attributed to the intercalation and deintercalation of electrolyte cations. Hence, $3 \mathrm{DNC} / \mathrm{MnO}_{x}$ shows better stability and reversible capacitive behavior.

In addition, electrochemical impedance spectroscopy (EIS) was also carried out to understand the electrode resistance and ion-transfer behavior of the electrode materials (Fig. 3f). The proposed equivalent circuit for the measured impedance data is shown in the inset of Fig. 3f, where $R_{\mathrm{S}}$ is the internal resistance, $R_{\mathrm{ct}}$ is faradic charge transfer resistance, CPE is the double-layer capacitance, and $Z_{\mathrm{w}}$ is the electrolyte resistance. ${ }^{2,9,20,35}$ Typically the semi-circle in medium frequency regions reflects the $R_{\mathrm{ct}}$. Before coating $\mathrm{MnO}_{x}$, the $R_{\mathrm{ct}}$ value of 3DNC $(23.6 \Omega)$ was much smaller than that of the $2 \mathrm{DNC}$ electrode $(65.5 \Omega)$. It should be attributed to the 3D network of 3DNC, which benefits the charge transfer between the electrode surface and the electrolyte. After coating $\mathrm{MnO}_{x}$, the $R_{\mathrm{ct}}$ of $3 \mathrm{DNC} / \mathrm{MnO}_{x}$ and $2 \mathrm{DNC} / \mathrm{MnO}_{x}$ increased to $54.9 \Omega$ and $82.8 \Omega$ due to the poor conductivity of $\mathrm{MnO}_{x}$. However, the $R_{\mathrm{ct}}$ value of $3 \mathrm{DNC} / \mathrm{MnO}_{x}$ was still much smaller than that of the $2 \mathrm{DNC} / \mathrm{MnO}_{x}$ electrode.

In the low frequency region, the slope of the curve represents the $Z_{\mathrm{w}}$, which is related to electrolyte diffusion in the porous electrode and proton diffusion in the active materials. ${ }^{35}$ As shown in Table 1 , the $Z_{\mathrm{w}}$ value of $3 \mathrm{DNC}(11.7 \Omega)$ and $3 \mathrm{DNC} /$ $\mathrm{MnO}_{x}(11.9 \Omega)$ were smaller than those of the 2DNC $(15.3 \Omega)$ and $2 \mathrm{DNC} / \mathrm{MnO}_{x}(20.3 \Omega)$ electrode, respectively. Those results were attributed to the unique hierarchical 3D structure based on $\mathrm{Si}$ pillar structure, which provided better electrolyte ion diffusion during fast charge and discharge processes.

In conclusion, we coaxially deposited $\mathrm{MnO}_{x}$ on a silicon pillar assisted 3DNC using an ECD process for supercapacitor application. The $C_{\mathrm{sp}}$ of $3 \mathrm{DNC} / \mathrm{MnO}_{x}$ is about $294 \mathrm{~F} \mathrm{~g}^{-1}$ at $10 \mathrm{mV}$ $\mathrm{s}^{-1}$ and the capacitance retention after 2000 cycles is as high as $96 \%$. The 3DNC structure provides not only a better ion and electron transfer pathway but also larger contact area and voids for electrolyte ion diffusion during fast charge and discharge processes. This unique nanostructure contributes to fast and reversible supercapacitor applications. 


\section{Acknowledgements}

The authors thank the National Research Foundation of Korea for financial support (2012M3A7B4035286, 2012R1A6A1029029, and 2016K1A4A3914691). Funding was also provided by a g-rant (AOARD-124044) from the AFOSR/AOARD, USA. S.

\section{References}

1 M. Toupin, T. Brousse and D. Belanger, Chem. Mater., 2004, 16, 3184-3190.

2 X. Xie and L. Gao, Carbon, 2007, 45, 2365-2373.

3 H. Zhang, G. P. Cao, Z. Y. Wang, Y. S. Yang, Z. J. Shi and Z. N. Gu, Nano Lett., 2008, 8, 2664-2668.

4 H. Xia, J. K. Feng, H. L. Wang, M. O. Lai and L. Lu, J. Power Sources, 2010, 195, 4410-4413.

5 W. Wei, X. Cui, W. Chen and D. G. Ivey, Chem. Soc. Rev., 2011, 40, 1697-1721.

6 J. Jiang, Y. Li, J. Liu, X. Huang, C. Yuan and X. W. Lou, Adv. Mater., 2012, 24, 5166-5180.

7 M. Zhi, C. Xiang, J. Li, M. Li and N. Wu, Nanoscale, 2013, 5, 72-88.

8 B. Brown, I. A. Cordova, C. B. Parker, B. R. Stoner and J. T. Glass, Chem. Mater., 2015, 27, 2430-2438.

9 M. Huang, X. L. Zhao, F. Li, L. L. Zhang and Y. X. Zhang, J. Power Sources, 2015, 277, 36-43.

10 J. Park, B. Kang, B. Kim, J.-S. Suh, Y.-M. Huh and S. Haam, Nano Convergence, 2015, 2, 1-8.

11 X. Lu, D. Zheng, T. Zhai, Z. Liu, Y. Huang, S. Xie and Y. Tong, Energy Environ. Sci., 2011, 4, 2915.

12 L. Zhang, K. N. Hui, K. S. Hui and H. Lee, Electrochim. Acta, 2015, 186, 522-529.

13 Y. Zhang, L. A. Huff, A. A. Gewirth and K. S. Suslick, Part. Part. Syst. Charact., 2015, 32, 899-906.

14 L. Zhang, K. N. Hui, K. S. Hui, X. Chen, R. Chen and H. Lee, Int. J. Hydrogen Energy, 2016, 41, 9443-9453.

15 L. Zhang, K. N. Hui, K. San Hui and H. Lee, J. Power Sources, 2016, 318, 76-85.

16 G. Nie, X. Lu, J. Lei and C. Wang, Electrochim. Acta, 2015, 180, 1033-1040.
17 R. R. Salunkhe, H. Ahn, J. H. Kim and Y. Yamauchi, Nanotechnology, 2015, 26, 204004.

18 Y. S. Moon, D. Kim, G. Lee, S. Y. Hong, K. K. Kim, S. M. Park and J. S. Ha, Carbon, 2015, 81, 29-37.

19 C. H. Ng, H. N. Lim, Y. S. Lim, W. K. Chee and N. M. Huang, Int. J. Energy Res., 2015, 39, 344-355.

20 Y.-Y. Horng, Y.-C. Lu, Y.-K. Hsu, C.-C. Chen, L.-C. Chen and K.-H. Chen, J. Power Sources, 2010, 195, 4418-4422.

21 S. J. Zhu, J. Zhang, J. J. Ma, Y. X. Zhang and K. X. Yao, J. Power Sources, 2015, 278, 555-561.

22 J. Maier, Nat. Mater., 2005, 4, 805-815.

23 A. L. M. Reddy, S. R. Gowda, M. M. Shaijumon and P. M. Ajayan, Adv. Mater., 2012, 24, 5045-5064.

24 M. Park, E. Oh, J. Seo, M. H. Kim, H. Cho, J. Y. Choi, H. Lee and I. S. Choi, Small, 2016, 12, 1148-1152.

25 S. Lee, J. Lee, D. W. Lee, J. M. Kim and H. Lee, Chem. Commun., 2016, 52, 926-929.

26 K. G. Lee, S. Lee, S. J. Chang, B. G. Choi, J. Seo, A. Sangalang, H. Kim do, T. J. Park, M. K. Lee, S. J. Lee and H. Lee, Small, 2015, 11, 4292-4297.

27 Y. D. Jo, S. Lee, J. Seo, S. Lee, D. Ann and H. Lee, J. Nanosci. Nanotechnol., 2014, 14, 9148-9151.

28 J. Seo, T. J. Lee, S. Ko, H. Yeo, S. Kim, T. Noh, S. Song, M. M. Sung and H. Lee, Adv. Mater., 2012, 24, 1975-1979.

29 M. K. Lee, J. Seo, S. J. Cho, Y. Jo, S. Kim, Y. Kang and H. Lee, Mater. Lett., 2012, 81, 9-12.

30 J. Seo, T. J. Lee, C. Lim, S. Lee, C. Rui, D. Ann, S. B. Lee and H. Lee, Small, 2015, 11, 2990-2994.

31 Y. Huang, A. R. Ferhan, S. J. Cho, H. Lee and D. H. Kim, ACS Appl. Mater. Interfaces, 2015, 7, 17582-17586.

32 C. D. Wagner and G. E. Muilenberg, Handbook of x-ray photoelectron spectroscopy: a reference book of standard data for use in $x$-ray photoelectron spectroscopy, Physical Electronics Division, Perkin-Elmer Corp., 1979.

33 M. Pourbaix and M. Pourbaix, published 1974 by NACE, 1974, vol. 644.

34 B. R. Strohmeier and D. M. Hercules, J. Phys. Chem., 1984, 88, 4922-4929.

35 C. Xia, Y. B. Xie, H. X. Du and W. Wang, J. Nanopart. Res., 2015, 17, 30 . 